\title{
Bone Union Assessment with Computed Tomography (CT) and Statistical Associations with Mechanical or Histological Testing: A Systematic Review of Animal Studies
}

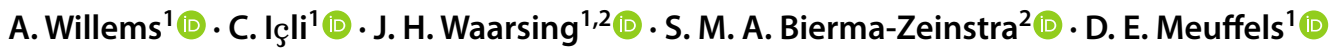

Received: 8 April 2021 / Accepted: 9 August 2021 / Published online: 21 August 2021

(c) The Author(s) 2021

\begin{abstract}
Objective and accurate assessment of bone union after a fracture, arthrodesis, or osteotomy is relevant for scientific and clinical purposes. Bone union is most accurately imaged with computed tomography (CT), but no consensus exists about objective assessment of bone union from CT images. It is unclear which CT-generated parameters are most suitable for bone union assessment. The aim of this review of animal studies is to find which CT-generated parameters are associated most strongly with actual bone union. Scientific databases were systematically searched. Eligible studies were studies that (1) were animal studies, (2) created a fracture, (3) assessed bone union with CT, (4) performed mechanical or histological testing as measure of actual bone union, and (5) associated CT-generated outcomes to mechanical or histological testing results. Two authors selected eligible studies and performed risk of bias assessment with QUADAS-2 tool. From 2567 studies that were screened, thirteen studies were included. Most common CT parameters that were investigated were bone mineral density, bone volume, and total callus volume. Studies showed conflicting results concerning the associations of these parameters with actual bone union. CT-assessed torsional rigidity (assessed by three studies) and callus density (assessed by two studies) showed best results. The studies investigating these two parameters reported moderate to strong associations with actual bone union. CT-assessed torsional rigidity and callus density seem the most promising parameters to represent actual bone union after a fracture, arthrodesis, or osteotomy.
\end{abstract}

Prospero trial registration number: CRD42020164733

Keywords Bone union · Computed tomography $\cdot$ Torsional test $\cdot$ Three-point bending test $\cdot$ Histological test $\cdot$ Torsional rigidity

\section{Introduction}

Achieving bone union is the main goal in patients after a fracture, osteotomy, or arthrodesis. But when has bone healed? This is a simple question, but the answer is rather complicated.

In the clinic, bone union is generally assessed based on conventional radiographs and on clinical examination, such

A. Willems

a.willems@erasmusmc.nl

1 Department of Orthopaedics and Sports Medicine, Erasmus MC University Medical Centre, Doctor Molewaterplein 40, 3015GD Rotterdam, The Netherlands

2 Department of General Practice, Erasmus MC University Medical Centre, Rotterdam, The Netherlands as response to weight bearing or palpation of the fracture [1]. However, assessing bone union is a rather subjective decision [2], and the lack of consensus has been extensively described by several studies [3, 4].

Assessment of bone union after a fracture, arthrodesis, or osteotomy is an important clinical consideration. Wrong assessment of bone healing can have major negative consequences for a patient. By overestimating the amount of bone healing, a bone might be loaded too early resulting in a displaced fracture or failure of osteosynthesis material. Underestimating bone healing may cause unnecessary immobilization resulting in stiffness, decreased muscle mass and function, and productivity loss of the patient $[5$, 6]. Especially if bone union is doubtful, an objective and accurate assessment tool can be helpful in clinical decisionmaking. Also, for scientific purposes, an objective and accurate method of fusion assessment would be of high value. 
Being able to accurately assess bone union would have several advantages like a decreased risk of biases within studies and less patients needed in clinical trials with bone union as primary outcome. Additionally, it would become easier to compare results between studies. In orthopedic studies, bone union is a commonly used primary outcome, for instance in studies investigating bone healing stimulating therapies after a fracture, osteotomy, or arthrodesis [7-9]. For the objective assessment of bone healing from radiographs, the radiographic union score (RUS) has been introduced in 2012 $[10,11]$. Ever since, this semi-quantitative assessment tool for assessment of fracture healing has become increasingly popular as an outcome measure in clinical studies $[12,13]$. However, computed tomography (CT) is the best method to image bone and has been shown to be superior to plain radiographs, MRI, and DEXA to assess bone union [14-17]. For CT, no golden standard exists for the objective assessment of bone union as an outcome measure. Therefore, we would like to create a method to objectively assess bone union from CT. This could then be used as golden standard for bone union assessment in clinical studies, but could also be used in the clinic if bone fusion after fracture, arthrodesis, or osteotomy is doubtful.

To establish an objective clinically applicable tool for bone union assessment, we need to know which CT-generated outcomes have a strong association with actual bone union. This review will therefore investigate which CT parameters are associated with actual bone healing. Actual bone union will be tested by mechanical or histological tests. As it is unethical and therefore impossible to acquire this data in clinical studies, in this review, we focus on animal studies. The aim of this review is to find CT parameters that best represent actual bone union, which is indicated by mechanical or histological testing.

\section{Method}

The protocol of this review has been prospectively registered at the International prospective register of systematic reviews (http://www.crd.york.ac.uk/prospero/; registration number CRD42020164733).

To find all studies concerning the assessment of bone union with $\mathrm{CT}$, an online search was performed on February 5, 2020. Five online databases were searched (Embase.com, Medline Ovid, Web of science, Cochrane CENTRAL, and Google Scholar). The search strategy for Medline Ovid is presented in Table 1 and was adapted for the other databases. Following the selection of eligible articles, reference lists of eligible articles were checked for missed articles.

After the search of the databases, eligible articles were selected, by two authors (AW and CI), based on predefined eligibility criteria (Table 2). Overall, we included studies that created a fracture in the appendicular skeleton of an animal. A fracture was defined as a bone gap that was created by performing an osteotomy or by impact loading.

Table 1 Search strategy for Medline Ovid

(fracture healing/ OR Fractures, Ununited/ OR (((bone* OR fracture* OR arthrodes* OR osteotom* OR scaphoid* OR osseous OR bony) ADJ6 (healing OR union* OR nonunion* OR united OR ununited OR consolidation))).ab,ti.) AND (exp "Validation Studies"/ OR "Comparative Study"/ OR exp "psychometrics"/ OR "outcome assessment (health care)"/ OR exp "observer variation"/ OR exp "Health Status Indicators"/ OR exp "reproducibility of results"/ OR exp "discriminant analysis"/ OR (psychometr* OR clinimetr* OR clinometr* OR (outcome ADJ3 (assessment* OR measure*)) OR (observer* ADJ3 variation*) OR ((reproducib* OR reliab* OR unreliab* OR valid* OR coefficient OR homogeneity OR homogeneous OR generaliza* OR generalisa* OR concordance OR repeatab* OR discriminative OR known group OR subscale* OR sensitiv* OR responsive* OR error OR errors) ADJ6 (diagnos* OR observ* OR tomograph* OR radiodiagnos* OR radiograph* OR x-ray*)) OR ((dimension*) ADJ6 (diagnos* OR observ* OR tomograph* OR radiodiagnos* OR radiograph* OR x-ray*) NOT (3-dimension* OR three-dimension*)) OR (internal* ADJ3 consisten*) OR (cronbach* ADJ3 (alpha OR alphas)) OR (item ADJ3 (correlation* OR selection* OR reduction*)) OR agreement OR precision OR imprecision OR (precise* ADJ3 value*) OR (test ADJ3 retest) OR (reliab* ADJ3 (test OR retest)) OR interrater* OR inter-rater* OR intrarater* OR intra-rater* OR intertester* OR inter-tester* OR intratester* OR intra-tester* OR interobserver* OR inter-observer* OR intraobserver* OR intra-observer* OR intertechnician* OR inter-technician* OR intratechnician* OR intra-technician* OR interexaminer* OR inter-examiner* OR intraexaminer* OR intra-examiner* OR interassay* OR inter-assay* OR intraassay* OR intra-assay* OR interindividual* OR inter-individual* OR intraindividual* OR intra-individual* OR interparticipant* OR inter-participant* OR intraparticipant* OR intra-participant* OR kappa OR kappa-s OR kappas OR ((replicab* OR repeated) ADJ3 (measure OR measures OR findings OR result OR results OR test OR tests)) OR (intraclass ADJ3 correlation*) OR (factor ADJ (analys* OR structure*)) OR (multitrait ADJ3 scaling ADJ3 (analysis OR analyses)) OR item discriminant OR (interscale ADJ3 correlation*) OR ((individual OR interval OR rate OR analysis OR values) ADJ3 variabil*) OR (uncertainty ADJ3 (measurement OR measuring)) OR standard error of measurement OR (limit ADJ3 detection) OR minimal detectable concentration OR interpretab* OR ((minimal OR minimally OR clinical OR clinically) ADJ3 (important OR significant OR detectable) ADJ3 (change OR difference)) OR (small* ADJ3 (real OR detectable) ADJ3 (change OR difference)) OR meaningful change OR ceiling effect OR floor effect OR Item response model OR Rasch OR Differential item functioning OR computer adaptive testing OR item bank OR cross-cultural equivalence OR ((defin* OR assess*) ADJ3 quanti*) OR (classif* ADJ3 (union OR consolidat*)) OR (union ADJ3 Score*)).ab,ti.) AND (exp Tomography, X-Ray Computed/ OR exp radiography/ OR Arthrography/ OR Diagnostic Imaging/ OR X ray film/ OR exp radiologists/ OR ((compute* ADJ3 tomograph*) OR radiodiagnos* OR radiolog* OR radiograph* OR x-ray* OR ct OR (cat ADJ (scan*)) OR rontgen* OR roentgen* OR microCT OR ((bone* OR diagnos*) ADJ3 imaging)).ab,ti.) NOT (letter OR news OR comment OR editorial OR congresses OR abstracts).pt. NOT (case reports/ OR case report.ti.) 
Table 2 In- and ex-clusion criteria

\begin{tabular}{ll}
\hline Inclusion & Exclusion \\
\hline - Animal study & $\bullet$ Bony defects or Distraction osteogenesis \\
- Bone fracture of the appendicular skeleton & \\
- Aim of the study to quantify bony union with micro CT, quantitative CT, multidetector CT, cone & $\bullet$ Follow-up period $<4$ weeks \\
beam CT, or clinical CT & $\bullet$ Data have been published before \\
- The relation between CT and histological or mechanical testing is statistically assessed & $\bullet$ Review article \\
- Article in English, Spanish, German, or Dutch & $\bullet$ Full text not available
\end{tabular}

Studies with distraction osteogenesis or bony defects were excluded. Bony defects were defined as drilling a hole in a bone. After at least 4 weeks, CT should be performed to assess bone union. The time period of 4 weeks was chosen because we aim to look at more advanced fracture healing and are not interested in the very early stages of bone healing. Simultaneously with CT, actual bone union should be tested by mechanical or histological testing. Parameters that are obtained from mechanical or histological testing and reflect bone union could be, for instance, bone mineral density, bone volume, or cross-sectional area. The association between CT outcomes and mechanical or histological outcomes should thereafter be statistically examined.

Firstly, based on the predefined in- and ex-clusion criteria, the eligibility of studies was assessed by reading title and abstract. Secondly, both authors read the full text of the pre-selected studies and assessed eligibility. After the first and second round, the study selection of both authors was compared. In case of disagreements, a third reviewer decided (DM).

Data were extracted from eligible studies using a predefined data extraction sheet. Data extraction was performed by one reviewer (AW) and checked by a second reviewer (CI). Disagreements were resolved by reaching consensus. Data that were extracted from the studies were data related to the methodology of the studies (fracture site, number of animals, animal species, use of bone growth stimulating injections, time till CT, type of CT, CT settings, volume of interest, threshold for bone, performance of histological testing and mechanical testing, mechanical test that was performed), outcome measures (outcomes of mechanical or histological testing, and outcomes of CT), and statistical associations between CT-outcomes and mechanical or histological testing.

Risk of bias assessment was done with the QUADAS-2 tool [18], which is a tool for diagnostic studies. Although the tool was originally designed for human studies, we chose this tool because it is the best available tool to assess risk of bias for studies in this review. The risk of bias assessment was done by two authors (AW and CI), and discrepancies were resolved by reaching consensus.
The primary outcome of this systematic review will be the strength of the associations between CT-assessed outcomes and mechanical or histological tested bone union. These associations can be expressed as Pearson's correlation coefficients, coefficients of determination, or strength of association in a regression model. To improve readability of this review, all linear Pearson's correlation coefficients will be squared, resulting in coefficients of determination. To distinguish between weak and strong relations, coefficients of determination will be classified as weak $\left(\mathrm{R}^{2}<0.4\right)$, moderate $\left(R^{2}=0.4-0.7\right)$, and strong $\left(R^{2}>0.7\right)$.

\section{Results}

The search initially resulted in 5159 studies. After removing the duplicates, 2567 studies were screened on title and abstract, resulting in 38 potentially eligible studies. After reading the full-text of those studies, thirteen studies were included in our systematic review (Fig. 1).

The results of the risk of bias assessment with the QUADAS-2 tool are presented in Table 3. The assessment showed that risk of bias is generally low in the domains 'animal selection' and 'flow and timing.' However, twelve studies did not clearly describe whether results of the index test (CT) were interpreted without the knowledge of the results of the reference test (mechanical or histological testing) and vice versa. Therefore, the risk of bias concerning these domains is unclear.

\section{General Study Characteristics}

The studies created a fracture by performing an osteotomy (eight times) [17, 19-25] or by impact loading (five times) [26-30]. Six studies created the fracture in the femur [19, 22, $26,27,29,30]$, six in the tibia $[17,20,21,24,25,28]$, and one in the metatarsus [23]. During follow-up, eight studies used micro-CT for the assessment of fracture healing [19, 22, 24-27, 29, 30], two studies peripheral quantitative CT [20, 21], and three studies (quantitative) clinical CT [17, 23, 28]. All studies performed mechanical testing, such as 

selection
Fig. 1 Flow chart of study

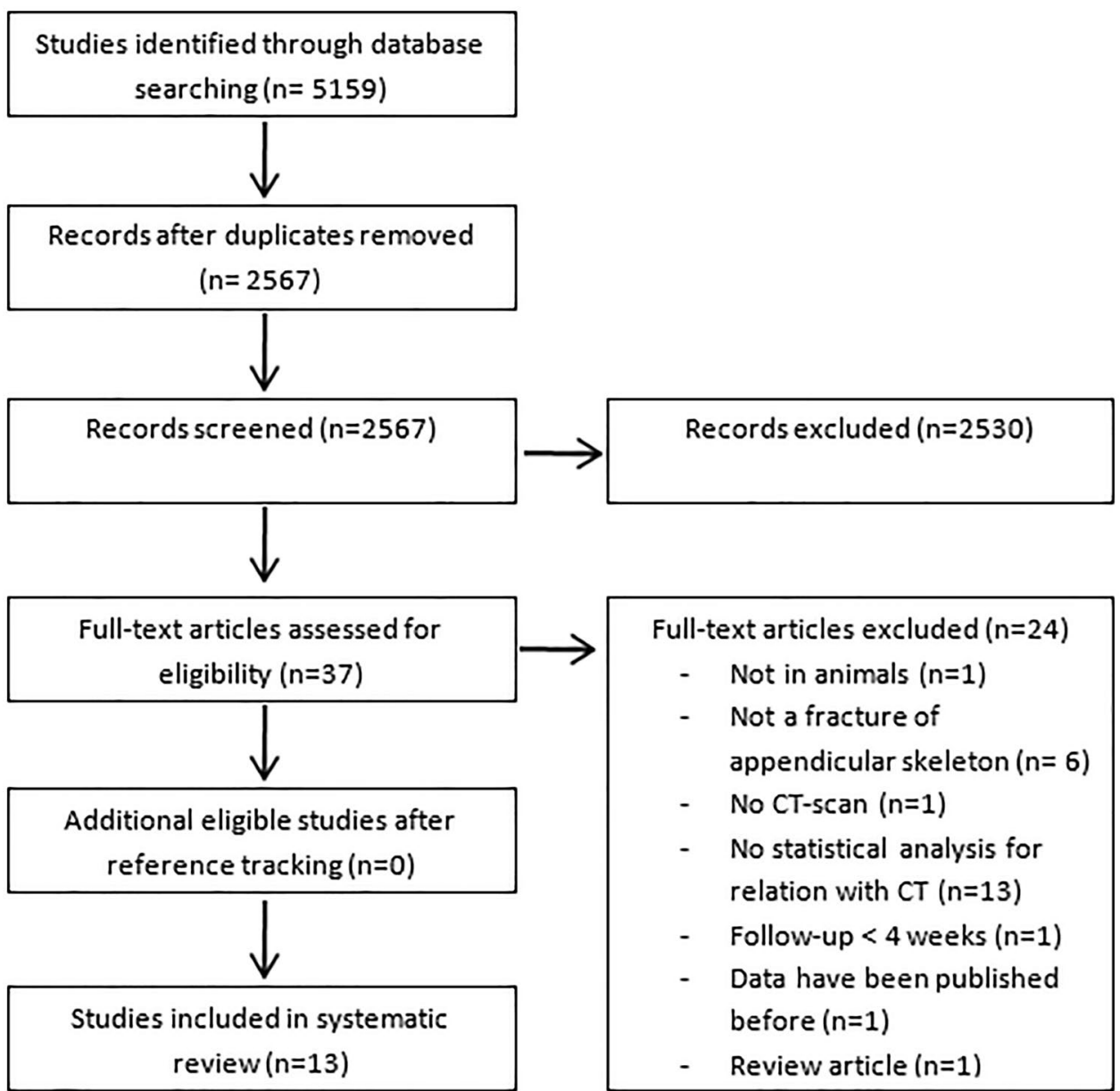

Table 3 Risk of bias assessment with the QUADAS-2 tool

\begin{tabular}{|c|c|c|c|c|c|c|c|}
\hline \multirow[t]{2}{*}{ Study } & \multicolumn{4}{|c|}{ Risk of bias } & \multicolumn{3}{|c|}{ Applicability concerns } \\
\hline & $\begin{array}{l}\text { Patient } \\
\text { selection }\end{array}$ & Index test & $\begin{array}{l}\text { Reference } \\
\text { standard }\end{array}$ & $\begin{array}{l}\text { Flow and } \\
\text { timing }\end{array}$ & $\begin{array}{l}\text { Patient } \\
\text { selection }\end{array}$ & Index test & $\begin{array}{l}\text { Refer- } \\
\text { ence } \\
\text { standard }\end{array}$ \\
\hline Mehta (2013) [29] & ;) & ;) & ;) & (;) & ;) & ;) & ;) \\
\hline Morgan (2009) [27] & ;) & $?$ & $?$ & ;) & ;) & ;) & ;) \\
\hline Nyman (2009) [26] & ;) & $?$ & $?$ & ;) & ;) & ;) & ;) \\
\hline Shefelbine (2005) [30] & ;) & $?$ & $?$ & ;) & ;) & ;) & ;) \\
\hline Nazarian (2010) [19] & ;) & $?$ & $?$ & ;) & ;) & ;) & ;) \\
\hline Fiset (2018) [22] & ;) & $?$ & $?$ & ;) & ;) & ;) & ;) \\
\hline Jämsä (2000) [21] & ;) & $?$ & $?$ & ;) & ;) & ;) & ;) \\
\hline Sigurdsen (2011) [25] & ;) & $?$ & $?$ & ;) & ;) & ;) & ;) \\
\hline Markel (1990) [17] & ;) & $?$ & $?$ & ;) & ;) & (:) & ;) \\
\hline Augat (1997) [20] & ;) & $?$ & $?$ & ;) & ;) & ;) & ;) \\
\hline Den Boer (1998) [28] & :) & $?$ & $?$ & (;) & ;) & ;) & ;) \\
\hline Wright (2012) [24] & ;) & $?$ & $?$ & (;) & ;) & ;) & ;) \\
\hline Böhm (1999) [23] & ;) & : & $?$ & ;) & ;) & ;) & ;) \\
\hline
\end{tabular}

-) Low risk, $\odot$ High risk, ? Unclear risk 
torsional tests $[17,19,22,24,27-30]$, three-point bending tests $[20,23,26]$, or axial tests $[17,21]$. Two studies also performed histological testing [20], but one of those did not correlate the outcomes to CT outcomes [17]. See Table 4 for animal species that were used and more study characteristics.

Linear relations between CT parameters and mechanical or histological outcomes were tested by performing Pearson's correlation $[21,22,25,26,29,30]$, bivariate linear regression [17, 19, 20, 23, 24, 28], or multiple regression analysis [27, 29]. Böhm and Jungkunz (1999) also performed bivariate quadratic regression analysis [23].

\section{Parameters Generated with CT Representing Bone Union}

\section{Quantitative CT Parameters}

Quantitative CT parameters that represent bone union are for example bone mineral density (BMD) and total volume of the callus (TV). Studies created volumes of interests (VOI) around the fracture, in which quantitative CT parameters were assessed. Table 5 shows the volumes of interests, bone thresholds, and outcome measures that were reported from CT. Also, it shows the parameters assessed from mechanical and histological testing.

\section{Biomechanical CT Parameters}

Three studies calculated the polar moment of inertia from CT $[23,27,30]$. Polar moment of inertia represents the resistance of bone to torsion and is dependent on the shape of the callus relative to the torsion axis. Polar moment of inertia is expressed as $\mathrm{m}^{4}$.

Three studies calculated torsional rigidity (GJ) of the fracture, based on CT-derived data $[19,24,30]$. GJ is a measure describing resistance of a bone when it is subjected to torsional forces and is expressed as $\mathrm{Nm}^{2}$. GJ is calculated from the cross-sectional area and CT-assessed bone mineral density. GJ was presented as an average of the entire VOI $\left(\mathrm{GJ}_{\mathrm{AVG}}\right)[19,24,30]$ and as the weakest slice of the VOI $\left(\mathrm{GJ}_{\mathrm{MIN}}\right)[19,24]$. Shefelbine et al. (2005) [30] also calculated the average bending rigidity.

Table 4 General study characteristics

\begin{tabular}{|c|c|c|c|c|c|c|c|c|}
\hline Study & $\begin{array}{l}\text { Fractured } \\
\text { bone }\end{array}$ & $\begin{array}{l}\text { Num- } \\
\text { ber of } \\
\text { animals }\end{array}$ & $\begin{array}{l}\text { Animal } \\
\text { species }\end{array}$ & $\begin{array}{l}\text { Bone- } \\
\text { stimulating } \\
\text { injection }\end{array}$ & $\begin{array}{l}\text { Time till CT } \\
\text { (weeks) }\end{array}$ & Type of CT & $\begin{array}{l}\text { Histo- } \\
\text { logical } \\
\text { testing }\end{array}$ & Mechanical testing \\
\hline $\begin{array}{l}\text { Mehta (2013) } \\
\text { [29] }\end{array}$ & Femur & 99 & Mice & No & $2-5$ & Micro & No & Torsional Testing \\
\hline $\begin{array}{l}\text { Morgan (2009) } \\
\text { [27] }\end{array}$ & Femur & 72 & Mice & Yes & $2-7$ & Micro & No & Torsional testing \\
\hline $\begin{array}{l}\text { Nyman }(2009) \\
{[26]}\end{array}$ & Femur & 53 & Rats & Yes & 4 & Micro & No & $\begin{array}{l}\text { Destructive three-point bend- } \\
\text { ing test }\end{array}$ \\
\hline $\begin{array}{l}\text { Shefelbine } \\
\text { (2005) [30] }\end{array}$ & Femur & 50 & Rats & No & 3,4 & Micro & No & Torsional Testing \\
\hline $\begin{array}{l}\text { Nazarian (2010) } \\
\text { [19] }\end{array}$ & Femur & 10 & Rats & Yes & 8 & Micro & No & Torsional testing \\
\hline $\begin{array}{l}\text { Fiset (2018) } \\
\text { [22] }\end{array}$ & Femur & 29 & Rats & No & $5-9$ or 17 & Micro & No & Torsional testing \\
\hline $\begin{array}{l}\text { Jämsä (2000) } \\
\text { [21] }\end{array}$ & Tibia & 141 & Rats & No & 4 or 8 & $\begin{array}{l}\text { Peripheral } \\
\text { quantitative }\end{array}$ & No & $\begin{array}{l}\text { Axial tension ( } 4 \text { weeks); axial } \\
\text { compression (after } 8 \text { weeks) }\end{array}$ \\
\hline $\begin{array}{l}\text { Sigurdsen } \\
\text { (2011) [25] }\end{array}$ & Tibia & 40 & Rats & No & $4,8.5$ & Micro & No & Bending test \\
\hline $\begin{array}{l}\text { Markel (1990) } \\
\text { [17] }\end{array}$ & Tibia & 32 & Dogs & No & $2,4,8$, or 12 & Quantitative & No & $\begin{array}{l}\text { Torsional testing and indenta- } \\
\text { tion testing }\end{array}$ \\
\hline $\begin{array}{l}\text { Augat (1997) } \\
\text { [20] }\end{array}$ & Tibia & 28 & Sheep & No & 9 & $\begin{array}{l}\text { Peripheral } \\
\text { quantitative }\end{array}$ & Yes & $\begin{array}{l}\text { Nondestructive three-point } \\
\text { bending test }\end{array}$ \\
\hline $\begin{array}{l}\text { Den Boer } \\
\text { (1998) [28] }\end{array}$ & Tibia & 24 & Goats & Yes & 2,4, or 6 & Axial spiral & No & Torsional testing \\
\hline $\begin{array}{l}\text { Wright (2012) } \\
\text { [24] }\end{array}$ & Tibia & 10 & Mice & No & 4 & Micro & No & Torsion testing \\
\hline $\begin{array}{l}\text { Böhm (1999) } \\
\text { [23] }\end{array}$ & Metatarsus & 12 & Sheep & No & 8 & Quantitative & No & $\begin{array}{l}\text { Nondestructive three-point } \\
\text { bending test }\end{array}$ \\
\hline
\end{tabular}




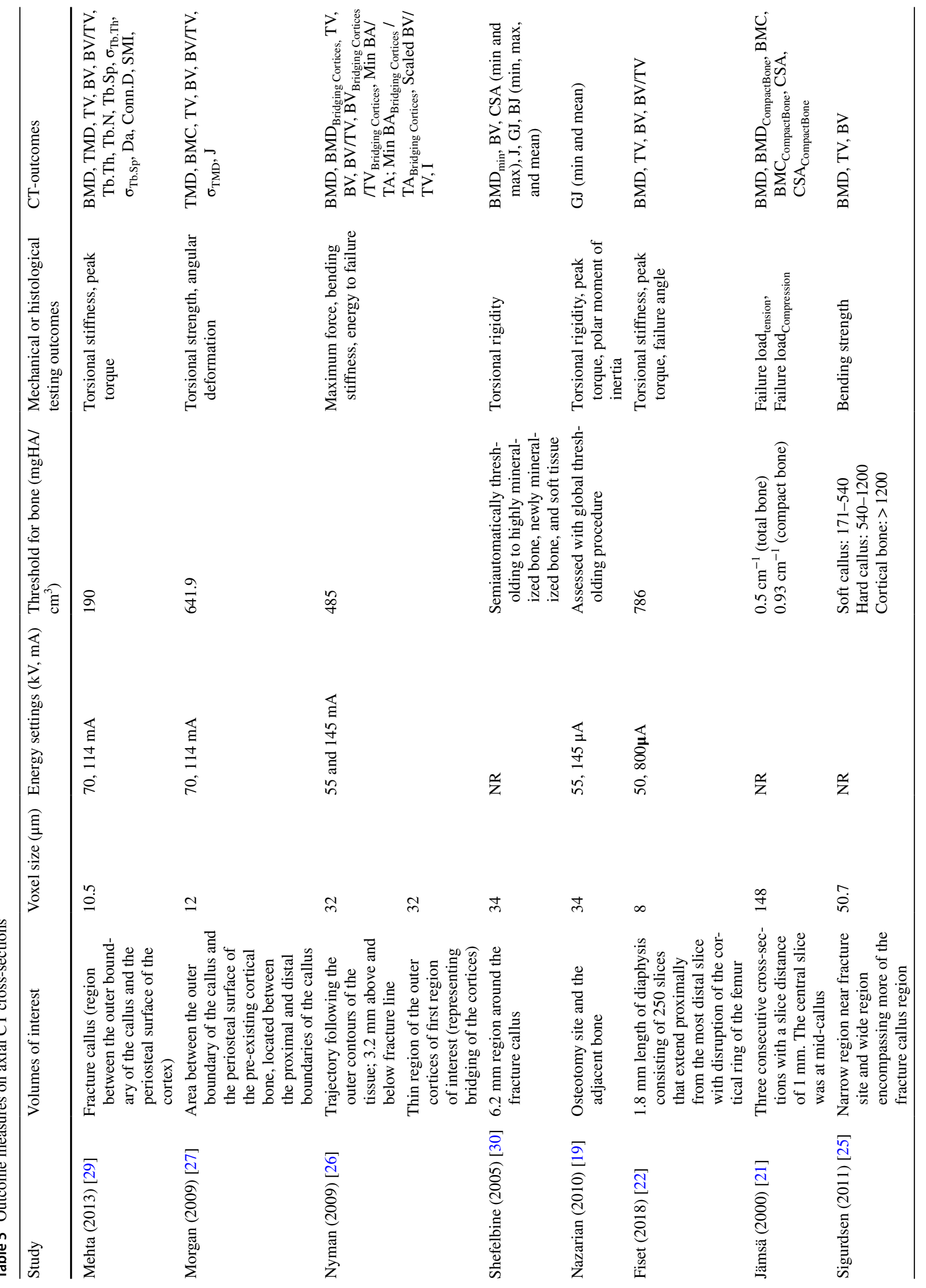




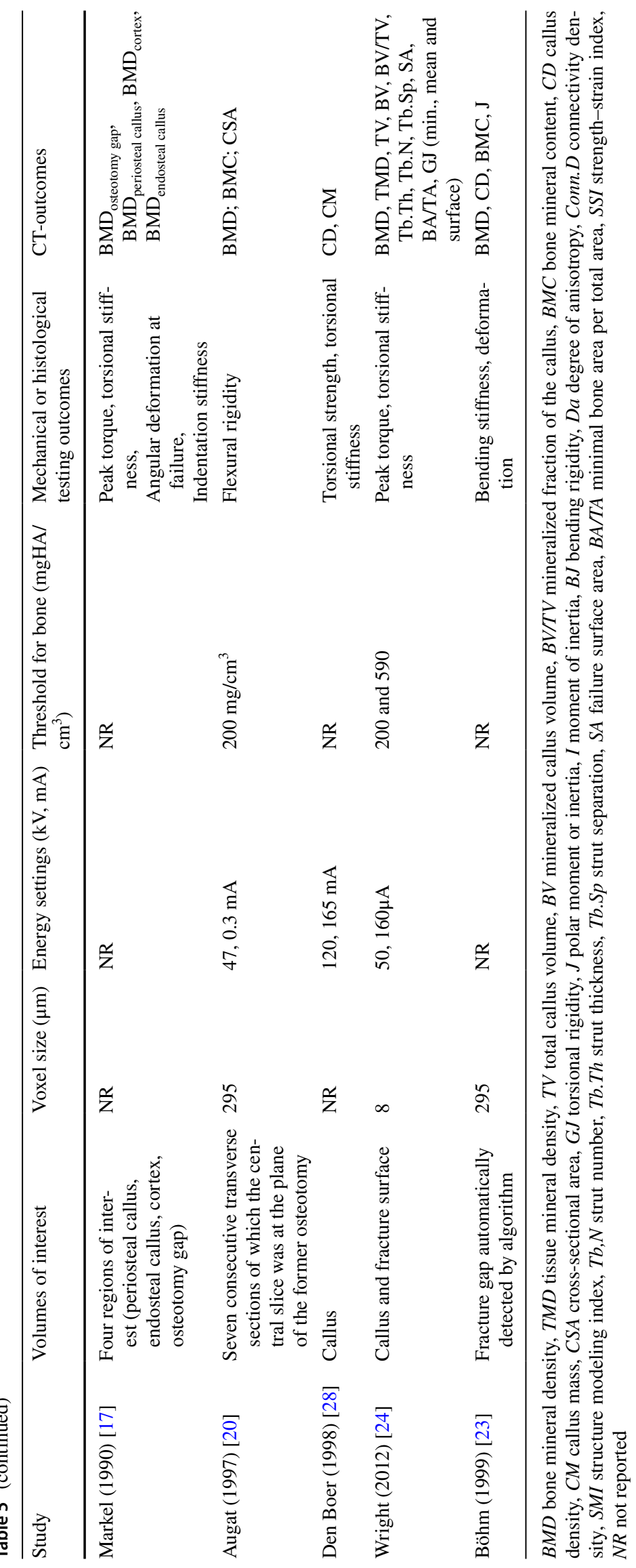




\section{Associations Between CT and Mechanical or Histological Testing}

\section{Quantitative CT Outcomes with Mechanical Testing}

The included studies used several quantitative parameters assessed from CT to represent bone union. The results of the studies are shown in Tables 6 and 7.

Ten studies correlated bone mineral density (BMD) to mechanical outcome. Six studies did not find associations with $\mathrm{R}^{2}>0.40$ between BMD and mechanical outcomes [21, 22, 24, 26, 29, 30]. Four studies found moderate to strong associations with BMD [17, 20, 23, 25]. Böhm and Jungkunz (1999) also found strong associations for a quadratic association between BMD and mechanical testing [23].

Callus density (CD) was assessed by two studies, which both reported strong associations between CD and mechanical testing [23, 28].

Tissue mineral density (TMD) was assessed by two studies [24, 29]. One study reported weak associations [29], whereas the other study found moderate associations between TMD and mechanical testing [24].

For bone mineral content (BMC), two studies did not find associations with $\mathrm{R}^{2}>0.40[20,21]$. One study reported a strong linear and quadratic association for BMC with mechanical testing [23].

Total callus volume (TV) was assessed by five studies. Three studies reported no or weak associations between TV and mechanical outcomes [25, 26, 29]. Two studies reported moderate associations with mechanical outcomes [22, 24].

Mineralized callus volume (BV) was assessed by six studies. Three studies reported no or weak associations for BV with mechanical outcomes [26, 29, 30]. Three studies reported moderate to strong associations between $\mathrm{BV}$ and mechanical outcomes [22, 24, 25].

The mineralized fraction of the callus (BV/TV) was assessed by four studies [22, 24, 26, 29], of which one study found a moderate association [26].

Cross-sectional area (CSA) was assessed by three studies and was not associated with mechanical outcomes [20, $21,30]$.

Some studies investigated less common CT-outcome parameters [24, 26-29]. From these parameters, associations with mechanical outcomes with $R^{2}>0.50$ were found for trabecular thickness [24] and amount of bone across the failure surface area [24].

Morgan et al. (2009) and Mehta, Heyland, Toben, and Duda (2013) created regression models to associate mechanical outcomes to quantitative CT parameters [27, 29]. For maximum torque, a model with TMD, BMC, and $\sigma \mathrm{TMD}$ explained $62 \%$ of the variation $\left(R^{2}=0.62\right)$, and a model with TMD, BV, and $\sigma$ TMD explained $61 \%$
$\left(R^{2}=0.61\right)$ [27]. For torsional rigidity, a model with TMD, BMC, BV/TV, and $\sigma \mathrm{TMD}$ explained $70 \%$ of the variation $\left(\mathrm{R}^{2}=0.70\right)$ [27]. Torsional stiffness could be predicted with a model containing strut thickness, the standard deviation of the strut separation, and strut number $\left(R^{2}=0.55\right)$. Torsional strength could be predicted with $\mathrm{BMD}$ or $\mathrm{BV} / \mathrm{TV}$, strut thickness, standard deviation, or strut separation $\left(R^{2}=0.57\right)$.

\section{Quantitative CT Outcomes with Histological Testing}

Augat et al. (1997) was the only study who correlated CToutcomes to histological outcomes. They reported a moderate association $\left(R^{2}=0.62\right)$ between minimal BMD and histologically assessed percentage bone in periosteal callus. A strong association $\left(R^{2}=0.71\right)$ was reported between the minimal BMD and histologically assessed percentage bone in fracture gap.

\section{Biomechanical CT Outcomes with Mechanical Testing}

Polar moment of inertia was assessed by three studies. Two studies found no or weak associations between moment of inertia and mechanical outcome [26, 30]. Böhm and Jungkunz (1999) reported moderate linear and quadratic associations between polar moment of inertia and mechanical testing [23].

Three studies associated $\mathrm{CT}$-assessed torsional rigidity to torsional rigidity assessed by mechanical testing [19, 24, 30]. All three studies reported moderate to strong associations between the average torsional rigidity and mechanical testing results $[19,24,30]$.

Shefelbine et al. (2005) reported moderate associations between $\mathrm{CT}$-assessed maximum and mean bending rigidity and mechanical outcomes [30].

\section{Data Synthesis}

Overall, for two parameters, all studies investigating these parameters found moderate or strong associations. These parameters were $\mathrm{CD}$, which was assessed by two studies, and CT-assessed torsional rigidity, which was assessed by three studies. For BMD, TMD, BMC, TV, BV, trabecular thickness, and polar moment of inertia, 30-60\% of the studies investigating these parameters found associations. For $\mathrm{BV} / \mathrm{TV}, \mathrm{CSA}$, trabecular number, trabecular separation, and bone area per total area, less than $30 \%$ of the studies found an association for these parameters.

Some parameters were only assessed by one study. From those, CT-assessed bending rigidity and amount of bone 
Table 6 Coefficients of determination for linear associations between CT-outcome measures and mechanical or histological testing outcomes

\begin{tabular}{|c|c|c|c|}
\hline \multirow[b]{2}{*}{ Bone mineral density (BMI } & \multicolumn{3}{|c|}{$\begin{array}{l}\text { Associations with mechanical or histological } \\
\text { testing }\end{array}$} \\
\hline & & & \\
\hline Mehta (2013) [29] & $.04^{\mathrm{B}}$ & $.04^{\mathrm{D}}$ & \\
\hline \multicolumn{4}{|l|}{ Nyman (2009) [26] } \\
\hline Bridging cortices & $.05^{\mathrm{G}}$ & $.05^{\mathrm{H}}$ & $<.01^{\mathrm{I}}$ \\
\hline Overall & $.20^{\mathrm{G}}$ & $.11^{\mathrm{H}}$ & $.03^{\mathrm{I}}$ \\
\hline \multicolumn{4}{|l|}{ Shefelbine (2005) [30] } \\
\hline Minimum & $.08^{\mathrm{J}}$ & & \\
\hline Fiset (2018) [22] & $.04^{\mathrm{B}}$ & $.22^{\mathrm{D}}$ & $.19^{\mathrm{E}}$ \\
\hline \multicolumn{4}{|l|}{ Jämsä (2000) [21] } \\
\hline Compact bone & $.09^{\mathrm{A} 1}$ & $.18^{\mathrm{A} 2}$ & \\
\hline Overall & $.04^{\mathrm{A} 1}$ & $.32^{\mathrm{A} 2}$ & \\
\hline \multicolumn{4}{|l|}{ Sigurdsen (2011) [25] } \\
\hline \multicolumn{4}{|l|}{30 days post-fracture } \\
\hline External fixator & $<.01^{\mathrm{F}}$ & & \\
\hline Intramedullary nail & $.68^{F}$ & & \\
\hline \multicolumn{4}{|l|}{60 days post-fracture } \\
\hline External fixator & $.25^{\mathrm{F}}$ & & \\
\hline Intramedullary nail & $.01^{\mathrm{F}}$ & & \\
\hline \multicolumn{4}{|l|}{ Markel (1990) [17] } \\
\hline Fracture gap & $.74^{B}$ & $.60^{D}$ & $.56^{K}$ \\
\hline Periosteal callus & $.73^{B}$ & $.49^{D}$ & $.64^{K}$ \\
\hline Cortex & n.s. ${ }^{\mathrm{B}}$ & n.s. ${ }^{\mathrm{D}}$ & n.s. ${ }^{\mathrm{K}}$ \\
\hline Endeosteal callus & n.s. ${ }^{B}$ & $.35^{\mathrm{D}}$ & $.40^{K}$ \\
\hline \multicolumn{4}{|l|}{ Augat (1997) [20] } \\
\hline Minimum & $.70^{\mathrm{L}}$ & $.62^{M}$ & $.71^{\mathrm{N}}$ \\
\hline \multicolumn{4}{|l|}{ Wright (2012) [24] } \\
\hline $0.2 \mathrm{gHa} / \mathrm{CM} 3$ & n.s. ${ }^{\text {B }}$ & n.s. ${ }^{\mathrm{D}}$ & \\
\hline $0.59 \mathrm{gHa} / \mathrm{CM} 3$ & n.s. ${ }^{\text {B }}$ & n.s. ${ }^{\text {D }}$ & \\
\hline \multicolumn{4}{|l|}{ Böhm (1999) [23] } \\
\hline Hard bone & $.70^{\mathrm{H}}$ & & \\
\hline Overall & $.75^{\mathrm{H}}$ & & \\
\hline \multicolumn{4}{|l|}{ Callus density (CD) } \\
\hline Den Boer (1998) [28] & $.82^{B}$ & $.72^{\mathrm{C}}$ & \\
\hline Böhm (1999) [23] & $.84^{\mathrm{H}}$ & & \\
\hline \multicolumn{4}{|c|}{ Tissue mineral density (TMD) } \\
\hline Mehta (2013) [29] & $.31^{\mathrm{B}}$ & $.28^{\mathrm{D}}$ & \\
\hline \multicolumn{4}{|l|}{ Wright (2012) [24] } \\
\hline $0.20 \mathrm{gHa} / \mathrm{CM}^{3}$ & $.60^{B}$ & n.s. ${ }^{\mathrm{D}}$ & \\
\hline $0.59 \mathrm{gHa} / \mathrm{CM}^{3}$ & $.63^{B}$ & n.s. ${ }^{\text {D }}$ & \\
\hline \multicolumn{4}{|c|}{ Bone mineral content (BMC) } \\
\hline \multicolumn{4}{|l|}{ Jämsä (2000) [21] } \\
\hline \multirow[t]{2}{*}{ Compact bone } & $.01^{\mathrm{A} 1}$ & & \\
\hline & $.32^{\mathrm{A} 2}$ & & \\
\hline \multirow[t]{2}{*}{ Overall } & $>.01^{\mathrm{A} 1}$ & & \\
\hline & $.20^{\mathrm{A} 2}$ & & \\
\hline Augat (1997) [20] & $.29^{\mathrm{L}}$ & & \\
\hline Böhm (1999) [23] & $.90^{\mathrm{H}}$ & & \\
\hline \multicolumn{4}{|l|}{ Total callus volume (TV) } \\
\hline Mehta (2013) [29] & $.07^{\mathrm{B}}$ & $.08^{\mathrm{D}}$ & \\
\hline Nyman 2009 & $.07^{\mathrm{G}}$ & $.18^{\mathrm{H}}$ & $.05^{\mathrm{I}}$ \\
\hline
\end{tabular}


Table 6 (continued) Associations with mechanical or histological testing

\begin{tabular}{|c|c|c|c|}
\hline Fiset (2018) [22] & $.56^{B}$ & $.45^{D}$ & $.30^{\mathrm{E}}$ \\
\hline \multicolumn{4}{|c|}{ Sigurdsen (2011) [25] } \\
\hline \multicolumn{4}{|c|}{$0.17-0.54 \mathrm{gHa} / \mathrm{CM}^{3} ; 30$ days post-fracture } \\
\hline External fixator & $.21^{\mathrm{F}}$ & & \\
\hline Intramedullary nail & $.26^{\mathrm{F}}$ & & \\
\hline \multicolumn{4}{|c|}{$0.17-0.54 \mathrm{gHa} / \mathrm{CM}^{3} ; 60$ days post-fracture } \\
\hline External fixator & $.10^{\mathrm{F}}$ & & \\
\hline Intramedullary nail & $.18^{\mathrm{F}}$ & & \\
\hline \multicolumn{4}{|c|}{$0.54-1.2 \mathrm{gHa} / \mathrm{CM}^{3} ; 30$ days post-fracture } \\
\hline External fixator & $.23^{\mathrm{F}}$ & & \\
\hline Intramedullary nail & $.12^{\mathrm{F}}$ & & \\
\hline \multicolumn{4}{|c|}{$0.54-1.2 \mathrm{gHa} / \mathrm{CM}^{3} ; 60$ days post-fracture } \\
\hline External fixator & $.01^{\mathrm{F}}$ & & \\
\hline Intramedullary nail & $.01^{\mathrm{F}}$ & & \\
\hline \multicolumn{4}{|l|}{ Wright (2012) [24] } \\
\hline $0.20 \mathrm{gHa} / \mathrm{CM}^{3}$ & $.54^{B}$ & n.s. ${ }^{\mathrm{D}}$ & \\
\hline $0.59 \mathrm{gHa} / \mathrm{CM}^{3}$ & $.54^{B}$ & n.s. ${ }^{\mathrm{D}}$ & \\
\hline \multicolumn{4}{|c|}{ Mineralized callus volume (BV) } \\
\hline Mehta (2013) [29] & $.08^{\mathrm{B}}$ & $.14^{\mathrm{D}}$ & \\
\hline Nyman 2009 & $.10^{\mathrm{G}}$ & $.07^{\mathrm{H}}$ & $<.01^{\mathrm{I}}$ \\
\hline Shefelbine (2005) [30] & $<.01^{\mathrm{J}}$ & & \\
\hline Fiset (2018) [22] & $.60^{B}$ & $.67^{D}$ & $.47^{E}$ \\
\hline \multicolumn{4}{|l|}{ Sigurdsen (2011) [25] } \\
\hline \multicolumn{4}{|l|}{30 days post-fracture } \\
\hline External fixator & $.17^{\mathrm{F}}$ & & \\
\hline Intramedullary nail & $.67^{F}$ & & \\
\hline \multicolumn{4}{|l|}{60 days post-fracture } \\
\hline External fixator & $.15^{\mathrm{F}}$ & & \\
\hline Intramedullary nail & $.19^{\mathrm{F}}$ & & \\
\hline \multicolumn{4}{|l|}{ Wright (2012) [24] } \\
\hline $0.20 \mathrm{gHa} / \mathrm{CM}^{3}$ & $.80^{B}$ & $.55^{D}$ & \\
\hline $0.59 \mathrm{gHa} / \mathrm{CM}^{3}$ & $.79^{B}$ & $.60^{D}$ & \\
\hline \multicolumn{4}{|c|}{ Mineralized fraction of the callus (BV/TV) } \\
\hline Mehta (2013) [29] & $.01^{\mathrm{B}}$ & $.01^{4}$ & \\
\hline \multicolumn{4}{|l|}{ Nyman 2009} \\
\hline Bridging cortices & $.20^{\mathrm{G}}$ & $.17^{\mathrm{H}}$ & $<.01^{\mathrm{I}}$ \\
\hline Overall & $<.01^{\mathrm{G}}$ & $.03^{\mathrm{H}}$ & $.12^{\mathrm{I}}$ \\
\hline Fiset (2018) [22] & $.27^{\mathrm{B}}$ & $.50^{D}$ & $.38^{\mathrm{E}}$ \\
\hline \multicolumn{4}{|l|}{ Wright (2012) [24] } \\
\hline $0.20 \mathrm{gHa} / \mathrm{CM}^{3}$ & n.s. ${ }^{B}$ & n.s. ${ }^{\mathrm{D}}$ & \\
\hline $0.59 \mathrm{gHa} / \mathrm{CM}^{3}$ & n.s. ${ }^{B}$ & n.s. ${ }^{\text {D }}$ & \\
\hline \multicolumn{4}{|l|}{ Cross-sectional area (CSA) } \\
\hline \multicolumn{4}{|l|}{ Shefelbine (2005) [30] } \\
\hline Minimum & $<.01^{\mathrm{J}}$ & & \\
\hline Maximum & $.01^{\mathrm{J}}$ & & \\
\hline \multicolumn{4}{|l|}{ Jämsä (2000) [21] } \\
\hline \multirow[t]{2}{*}{ Compact bone } & $.00^{\mathrm{A} 1}$ & & \\
\hline & $.16^{\mathrm{A} 2}$ & & \\
\hline \multirow[t]{2}{*}{ Overall } & $<.01^{\mathrm{A} 1}$ & & \\
\hline & $.04^{\mathrm{A} 2}$ & & \\
\hline
\end{tabular}


Table 6 (continued)

Associations with mechanical or histological testing

\begin{tabular}{|c|c|c|c|}
\hline Augat (1997) [20] & $.15^{\mathrm{L}}$ & & \\
\hline \multicolumn{4}{|l|}{ Callus mass (CM) } \\
\hline Den Boer (1998) [28] & $.05^{\mathrm{B}}$ & $.02^{\mathrm{C}}$ & \\
\hline \multicolumn{4}{|c|}{ Trabecular thickness (Tb.Th) } \\
\hline Mehta (2013) [29] & $.34^{\mathrm{B}}$ & $.32^{\mathrm{D}}$ & \\
\hline \multicolumn{4}{|l|}{ Wright (2012) [24] } \\
\hline $0.20 \mathrm{gHa} / \mathrm{CM}^{3}$ & n.s. ${ }^{B}$ & n.s. ${ }^{\mathrm{D}}$ & \\
\hline $0.59 \mathrm{gHa} / \mathrm{CM}^{3}$ & $.63^{B}$ & $.52^{D}$ & \\
\hline \multicolumn{4}{|l|}{ Trabecular number (Tb.N) } \\
\hline Mehta (2013) [29] & $.01^{\mathrm{B}}$ & $<.01^{\mathrm{D}}$ & \\
\hline \multicolumn{4}{|l|}{ Wright (2012) [24] } \\
\hline $0.20 \mathrm{gHa} / \mathrm{CM}^{3}$ & n.s. ${ }^{B}$ & n.s. ${ }^{\mathrm{D}}$ & \\
\hline $0.59 \mathrm{gHa} / \mathrm{CM}^{3}$ & n.s. ${ }^{B}$ & n.s. ${ }^{\mathrm{D}}$ & \\
\hline \multicolumn{4}{|c|}{ Trabecular separation (Tb.Sp) } \\
\hline Mehta (2013) [29] & $.02^{\mathrm{B}}$ & $.05^{\mathrm{D}}$ & \\
\hline \multicolumn{4}{|l|}{ Wright (2012) [24] } \\
\hline $0.20 \mathrm{gHa} / \mathrm{CM}^{3}$ & n.s. ${ }^{\text {B }}$ & n.s. ${ }^{\mathrm{D}}$ & \\
\hline $0.59 \mathrm{gHa} / \mathrm{CM}^{3}$ & n.s. ${ }^{B}$ & n.s. ${ }^{\mathrm{D}}$ & \\
\hline \multicolumn{4}{|l|}{ Standard deviation (Tb.Th) } \\
\hline Mehta (2013) [29] & $.31^{\mathrm{B}}$ & $.31^{\mathrm{D}}$ & \\
\hline \multicolumn{4}{|l|}{ Standard deviation (Tb.Sp) } \\
\hline Mehta (2013) [29] & $.14^{\mathrm{B}}$ & $.18^{\mathrm{D}}$ & \\
\hline \multicolumn{4}{|l|}{ Failure surface area (SA) } \\
\hline \multicolumn{4}{|l|}{ Wright (2012) [24] } \\
\hline Bone $0.2 \mathrm{gHa} / \mathrm{CM}^{3}$ & $.73^{B}$ & n.s. ${ }^{\mathrm{D}}$ & \\
\hline Bone $0.59 \mathrm{gHa} / \mathrm{CM}^{3}$ & $.62^{B}$ & $.59^{D}$ & \\
\hline Total $0.2 \mathrm{gHa} / \mathrm{CM}^{3}$ & n.s. ${ }^{\text {B }}$ & n.s. ${ }^{\mathrm{D}}$ & \\
\hline Total $0.59 \mathrm{gHa} / \mathrm{CM}^{3}$ & n.s. ${ }^{B}$ & n.s. ${ }^{\mathrm{D}}$ & \\
\hline \multicolumn{4}{|c|}{ Bone area per total area (BA/TA) } \\
\hline \multicolumn{4}{|l|}{ Nyman 2009} \\
\hline Bridging cortices & $.29^{\mathrm{G}}$ & $.21^{\mathrm{H}}$ & $<.01^{\mathrm{I}}$ \\
\hline Overall & $<.01^{\mathrm{G}}$ & $.06^{\mathrm{H}}$ & $.05^{\mathrm{I}}$ \\
\hline \multicolumn{4}{|l|}{ Wright (2012) [24] } \\
\hline $0.20 \mathrm{gHa} / \mathrm{CM}^{3}$ & n.s. ${ }^{B}$ & n.s. ${ }^{\mathrm{D}}$ & \\
\hline $0.59 \mathrm{gHa} / \mathrm{CM}^{3}$ & n.s. ${ }^{B}$ & n.s. ${ }^{\mathrm{D}}$ & \\
\hline \multicolumn{4}{|l|}{ Degree of anisotropy (DA) } \\
\hline Mehta (2013) [29] & $.02^{\mathrm{B}}$ & $.04^{\mathrm{D}}$ & \\
\hline \multicolumn{4}{|c|}{ Connectivity density (Conn.D) } \\
\hline Mehta 2013 & $.25^{\mathrm{B}}$ & $.15^{\mathrm{D}}$ & \\
\hline \multicolumn{4}{|c|}{ Structure modeling index (SMI) } \\
\hline Mehta (2013) [29] & $.10^{\mathrm{B}}$ & $.12^{\mathrm{D}}$ & \\
\hline \multicolumn{4}{|l|}{ Polar moment of inertia } \\
\hline \multicolumn{4}{|l|}{ Nyman 2009} \\
\hline \multicolumn{4}{|l|}{ Overall } \\
\hline Mean & $.08^{\mathrm{G}}$ & $.10^{\mathrm{H}}$ & $.00^{\mathrm{I}}$ \\
\hline Min & $<.01^{\mathrm{G}}$ & $.03^{\mathrm{H}}$ & $<.01^{\mathrm{I}}$ \\
\hline \multicolumn{4}{|l|}{ Bridging cortices } \\
\hline Mean & $<.02^{\mathrm{G}}$ & $.09^{\mathrm{H}}$ & $.07^{\mathrm{I}}$ \\
\hline Min & $<.01^{\mathrm{G}}$ & $.01^{\mathrm{H}}$ & $.09^{9}$ \\
\hline Shefelbine (2005) [30] & $.04^{\mathrm{J}}$ & & \\
\hline
\end{tabular}


Table 6 (continued)

\begin{tabular}{|c|c|c|}
\hline & \multicolumn{2}{|c|}{$\begin{array}{l}\text { Associations with mechanical or histological } \\
\text { testing }\end{array}$} \\
\hline \multicolumn{3}{|l|}{ Böhm (1999) [23] } \\
\hline Calculated from center of mass & \multicolumn{2}{|l|}{$.68^{J}$} \\
\hline Calculated from geometric midpoint & \multicolumn{2}{|l|}{$.69^{J}$} \\
\hline \multicolumn{3}{|l|}{$\mathrm{CT}$-assessed torsional rigidity } \\
\hline Shefelbine (2005) [30] & \multicolumn{2}{|l|}{$.48^{J}$} \\
\hline \multicolumn{3}{|l|}{ Nazarian 2010} \\
\hline Smallest & $.78^{\mathrm{D}}$ & $.81^{\mathrm{J}}$ \\
\hline Average & n.s. ${ }^{\mathrm{D}}$ & $.63^{J}$ \\
\hline \multicolumn{3}{|l|}{ Wright (2012) [24] } \\
\hline Smallest & n.s. ${ }^{B}$ & n.s. ${ }^{\mathrm{D}}$ \\
\hline Average & $.50^{B}$ & n.s. ${ }^{\mathrm{D}}$ \\
\hline Surface & $.66^{B}$ & n.s. ${ }^{\mathrm{D}}$ \\
\hline \multicolumn{3}{|l|}{ CT-assessed bending rigidity } \\
\hline \multicolumn{3}{|l|}{ Shefelbine (2005) [30] } \\
\hline Smallest & \multicolumn{2}{|l|}{$.49^{J}$} \\
\hline Largest & \multicolumn{2}{|l|}{$.52^{J}$} \\
\hline Mean & \multicolumn{2}{|l|}{$.52^{J}$} \\
\hline
\end{tabular}

Bold indicate strong $\left(R^{2}>0.7\right)$ associations; italic indicate moderate associations $\left(R^{2} 0.4-0.7\right)$; $A 1$ failure load tension; $A 2$ : failure load compression; $B$ torsional stiffness; $C$ torsional strength; $D$ peak torque; $E$ failure angle; $F$ bending strength; $G$ maximum force; $H$ Bending stiffness; $I$ Energy to failure; $J$ Torsional rigidity; $K$ Indentation stiffness; $L$ Flexural rigidity; $M$ histologically $\%$ bone in periosteal callus; $N$ histologically $\%$ bone in fracture gap; $T$ coefficient of determination for failure load under tension; $C$ coefficient of determination for failure load under compression; n.s. not significant, no coefficient of determination reported

across the failure surface area showed moderate to strong associations.

Table 7 Coefficients of determination for quadratic associations between CT-outcome measures and mechanical or histological testing outcomes

\begin{tabular}{ll}
\hline & $\begin{array}{l}\text { Outcome measures of } \\
\text { mechanical or histological } \\
\text { testing }\end{array}$ \\
\hline $\begin{array}{l}\text { Bone mineral density (BMD) } \\
\text { Böhm (1999) [23] }\end{array}$ & $\mathbf{. 7 4}$ \\
$\quad$ Hard bone & $\mathbf{. 7 6}^{\mathbf{H}}$ \\
Overall & \\
Callus density (CD) & $\mathbf{. 5 5}^{\mathbf{H}}$ \\
Böhm (1999) [23] & \\
Bone mineral content (BMC) & $\mathbf{. 9 3}^{\mathbf{H}}$ \\
Böhm (1999) [23] & \\
Polar moment of inertia & \\
Böhm (1999) [23] & $.68^{H}$ \\
Calculated from center of mass & $.69^{H}$ \\
Calculated from geometric midpoint
\end{tabular}

Bold indicate strong $\left(\mathrm{R}^{2}>0.7\right)$ associations; italic indicate moderate associations $\left(\mathrm{R}^{2}\right.$ 0.4-0.7) H: Bending stiffness

\section{Discussion}

We aimed to identify CT-outcome parameters which are associated most strongly with bone union after a fracture. The associations found by the studies are conflicting, with exception for $\mathrm{CT}$-assessed torsional and bending rigidity, and callus density.

CT-assessed torsional rigidity was found to have moderate to strong associations by all three studies that investigated it. Torsional rigidity is calculated from CT-acquired data and is dependent on the callus density, cross-sectional area, and the distribution of bone density within the callus $[19,30]$. Based on CT, virtual models of the bone are created on which virtual mechanical testing can be performed. From this virtual testing, torsional rigidity is calculated [19, 30 , 31]. Average torsional rigidity showed moderate associations with mechanical tests in all three studies [19, 24, 30]. The results of Naziarian et al. (2010) [19] showed that minimum torsional rigidity had a stronger association with mechanical testing than average torsional rigidity. This means that analyzing only the weakest segment (axial slice) of CT images would give the strongest associations. This seems logical, as failure of a beam under forces is dependent on the weakest point, and not the average strength [19]. However, Wright et al. (2012) [24] did not find an association 
between minimum torsional rigidity and mechanical testing. According to Wright, the use of the tibia, and not the femur as Nazarian did, might explain this [24]. In contrast to the femur, the diameter of the tibia decreases when going more distally. As torsional rigidity is dependent on the CSA, the minimum torsional rigidity might therefore move to the most distal part of the VOI when analyzing the tibia [24]. This once more indicates that the assessment of fracture healing is complex and dependent on many variables.

This complexity may have led to the conflicting results of the other parameters. For example, quite strong association were reported for BMD by three studies, whereas other studies found no associations with BMD. Because of the conflicting results between studies, the generalizability of the associations seems to be quite low. Also, most studies in this review explored linear relations, but Böhm and Jungkunz (1999) showed that associations might be quadratic [23]. However, Böhm and Jungkunz (1999) was the only study investigating quadratic associations and it was a small study $(n=12)$.

So far, CT-assessed torsional rigidity seems a promising parameter for bone union assessment. Clinically, several studies have been investigating CT-assessed torsional rigidity. CT-assessed torsional rigidity was successfully used for the prediction of fractures in patients with bone lesions [32-34]. Also, recently, the first clinical study has been published that used CT-assessed torsional rigidity to assess tibial fracture healing [35]. In this study, a low-dose CT was made of the tibia 12 weeks after surgical fixation. Software was used to create a virtual model of the fractured tibia which was adapted to a model of an intact tibia. Virtual torsional testing could then be performed on these models, resulting in torsional rigidity values for the fractured and intact tibia. Lastly, torsional rigidity of the fractured model was divided by the torsional rigidity of the intact model. By doing this, a dimensionless parameter was created which indicates the progression of healing relative to the intact tibia [31]. Given the results of this review, and the promising results of the first clinical study, CT-assessed torsional rigidity could become a useful tool for bone union assessment. However, at this moment, the clinical applicability of CTassessed torsional rigidity is limited. Advanced software and knowledge are needed to conduct CT-based structural rigidity analysis (CTRA) [32]. Although CTRA can be done with data from any CT-scanner, bone densities are very important for the analysis. Therefore, phantoms with known bone densities should be scanned with the patient [32].

This systematic review encountered some limitations. Firstly, CT-assessed torsional rigidity and callus density were only assessed by a limited number of studies (three studies for CT-assessed torsional rigidity and two studies for callus density). Although those studies show promising results, more studies should be done to further confirm these results. Parameters that were assessed by more than three studies had higher chances of finding contradictory results. However, the more investigated parameters in this review showed no significant associations in most of the studies. BMD for instance was investigated by ten studies, of which only four reported significant associations. A second limitation of this review is that the statistical associations that are presented come from animal studies. We should be careful by translating these results directly to clinical human fractures, as data retrieved from animal studies might be unreliable in clinical studies [10]. For example, studies have shown that rodent bone remodeling is different from large animal or human bone remodeling because it is lacking intracortical remodeling [36]. Therefore, associations for bone healing might be different for rodents compared to large animals or humans. Also, most studies in this review used micro CT-scanners with higher spatial resolutions and higher radiation doses than clinical CT-scanners [19, 21, 26, 27]. Therefore, clinical CT-scanners might be less accurate than micro CT-scanners [37]. Thirdly, for this systematic review, we used fairly strict inclusion criteria. The main reason for these strict criteria was to keep heterogeneity between studies as low as possible to be able to compare studies and therewith draw a firm conclusion. Even with these strict criteria, the heterogeneity between studies was high. Studies used different location of fractures, animal species, scanning protocols, and mechanical testing protocols, which is likely to affect the associations found between the studies. Also, four studies used drug treatments to increase fracture healing [19, 26-28]. These treatments can modulate structural and mechanical properties of the callus [38]. Due to the strict inclusion criteria, many studies were excluded during the study selection process. These were also studies who assessed bone healing by performing CT, mechanical and histological testing. However, in these studies, the different methods were used complementary to each other and the results of these methods were not compared to each other. Therefore, it is not possible to draw conclusions from these studies concerning the best CT-outcome parameter. Also, minimal follow-up time was set to 4 weeks, as we were not interested in studies who only looked at early stages of fracture healing. As fracture healing progresses differently between animal species and depends on fracture size, one could argue if this period was accurate. Also, the associations between CT parameters and mechanical and histological outcomes might be influenced by the stage of fracture healing, which may vary between the studies. Lastly, the risk of bias of studies was assessed with the QUADAS-2 tool. As this tool is designed for clinical studies, it may not be accurate for pre-clinical studies. However, no pre-clinical risk of bias tool exists for diagnostic studies. Most studies in this review showed concerns about risk of bias. To decrease risk of bias in future studies, we strongly recommend to 
interpret the index test (CT), without knowing the results of the reference test (mechanical or histological testing) and to describe this process in the paper.

Based on the currently available literature, density-related parameters seem to be most promising parameters to assess bone union after a fracture. Especially, CT-assessed torsional rigidity is a promising parameter to assess bone union. To improve the clinical assessment of fracture healing, we encourage the conduction of more high-quality clinical studies investigating the applicability of CT-assessed torsional rigidity for bone union assessment. In the future, torsional rigidity could potentially become a widely accepted outcome measure for bone union assessment in clinical studies and in clinical practice.

Acknowledgements Many thanks to Wichor Bramer, Biomedical information specialist at the Erasmus MC, for his help with the systematic search of the databases.

Author Contributions AW had the idea for the article, preformed literature search and data analysis, and wrote first draft of the article. CI performed literature search and data analysis. JHW performed the data analysis and critically supervised. SMABZ advised on methodology and critically supervised. DEM had the idea for the article and critically revised. All authors revised the paper critically for intellectual content and approved the final version. All authors agree to be accountable for the work and to ensure that any questions relating to the accuracy and integrity of the paper are investigated and properly resolved.

Funding No funding was received for conducting this study.

Data Availability Data available within the article.

\section{Declarations}

Conflict of interest A. Willems, C. Içli, J.H. Waarsing, S.M.A. Bierma-Zeinstra, D.E. Meuffels have no relevant financial or non-financial interest to disclose.

Open Access This article is licensed under a Creative Commons Attribution 4.0 International License, which permits use, sharing, adaptation, distribution and reproduction in any medium or format, as long as you give appropriate credit to the original author(s) and the source, provide a link to the Creative Commons licence, and indicate if changes were made. The images or other third party material in this article are included in the article's Creative Commons licence, unless indicated otherwise in a credit line to the material. If material is not included in the article's Creative Commons licence and your intended use is not permitted by statutory regulation or exceeds the permitted use, you will need to obtain permission directly from the copyright holder. To view a copy of this licence, visit http://creativecommons.org/licenses/by/4.0/.

\section{References}

1. Bhandari M, Fong K, Sprague S, Williams D, Petrisor B (2012) Variability in the definition and perceived causes of delayed unions and nonunions: a cross-sectional, multinational survey of orthopaedic surgeons. J Bone Joint Surg Am 94:e1091-1096. https://doi.org/10.2106/JBJS.K.01344

2. Bhandari M, Guyatt GH, Swiontkowski MF, Tornetta P 3rd, Sprague S, Schemitsch EH (2002) A lack of consensus in the assessment of fracture healing among orthopaedic surgeons. J Orthop Trauma 16:562-566. https://doi.org/10.1097/00005131200209000-00004

3. Cunningham BP, Brazina S, Morshed S, Miclau T 3rd (2017) Fracture healing: A review of clinical, imaging and laboratory diagnostic options. Injury 48(Suppl 1):S69-S75. https://doi.org/ 10.1016/j.injury.2017.04.020

4. Corrales LA, Morshed S, Bhandari M, Miclau T 3rd (2008) Variability in the assessment of fracture-healing in orthopaedic trauma studies. J Bone Joint Surg Am 90:1862-1868. https://doi.org/10. 2106/JBJS.G.01580

5. Dudley GA, Duvoisin MR, Adams GR, Meyer RA, Belew AH, Buchanan P (1992) Adaptations to unilateral lower limb suspension in humans. Aviat Space Environ Med 63:678-683

6. Bloomfield SA (1997) Changes in musculoskeletal structure and function with prolonged bed rest. Med Sci Sports Exerc 29:197206. https://doi.org/10.1097/00005768-199702000-00006

7. Willems A, van der Jagt OP, Meuffels DE (2019) Extracorporeal shock wave treatment for delayed union and nonunion fractures: a systematic review. J Orthop Trauma 33:97-103. https://doi.org/ 10.1097/BOT.0000000000001361

8. Bovbjerg P, Høgh D, Froberg L, Schmal H, Kassem M (2021) Effect of PTH treatment on bone healing in insufficiency fractures of the pelvis: a systematic review. EFORT Open Rev 6:9-14. https://doi.org/10.1302/2058-5241.6.200029

9. Van Lieshout EMM, Den Hartog D (2020) Effect of platelet-rich plasma on fracture healing. Injury. https://doi.org/10.1016/j.injury. 2020.12.005

10. Schwarzenberg P, Darwiche S, Yoon RS, Dailey HL (2020) Imaging modalities to assess fracture healing. Curr Osteoporos Rep 18:169-179. https://doi.org/10.1007/s11914-020-00584-5

11. Whelan DB, Bhandari M, Stephen D, Kreder H, McKee MD, Zdero R, Schemitsch EH (2010) Development of the radiographic union score for tibial fractures for the assessment of tibial fracture healing after intramedullary fixation. J Trauma 68:629-632

12. Leow JM, Clement ND, Simpson A (2020) Application of the Radiographic Union Scale for Tibial fractures (RUST): assessment of healing rate and time of tibial fractures managed with intramedullary nailing. Orthop Traumatol Surg Res 106:89-93

13. Litrenta J, Tornetta P 3rd, Mehta S, Jones C, O'Toole RV, Bhandari M, Kottmeier S, Ostrum R, Egol K, Ricci W, Schemitsch E, Horwitz D (2015) Determination of radiographic healing: an assessment of consistency using RUST and modified rust in metadiaphyseal fractures. J Orthop Trauma 29:516-520

14. Braunstein EM, Goldstein SA, Ku J, Smith P, Matthews LS (1986) Computed tomography and plain radiography in experimental fracture healing. Skeletal Radiol 15:27-31. https://doi.org/10. 1007/bf00355070

15. Firoozabadi R, Morshed S, Engelke K, Prevrhal S, Fierlinger A, Miclau T 3rd, Genant HK (2008) Qualitative and quantitative assessment of bone fragility and fracture healing using conventional radiography and advanced imaging technologies-focus on wrist fracture. J Orthop Trauma 22:S83-90. https://doi.org/10. 1097/BOT.0b013e31815ea2a4

16. Grigoryan M, Lynch JA, Fierlinger AL, Guermazi A, Fan B, MacLean DB, MacLean A, Genant HK (2003) Quantitative and qualitative assessment of closed fracture healing using computed tomography and conventional radiography. Acad Radiol 10:12671273. https://doi.org/10.1016/s1076-6332(03)00467-7

17. Markel MD, Wikenheiser MA, Morin RL, Lewallen DG, Chao EY (1990) Quantification of bone healing. Comparison of QCT, 
SPA, MRI, and DEXA in dog osteotomies. Acta Orthop Scand 61:487-498. https://doi.org/10.3109/17453679008993569

18. Whiting PF, Rutjes AWS, Westwood ME, Mallett S, Deeks JJ, Reitsma JB, Leeflang MMG, Sterne JAC, Bossuyt PMM, the Q-G (2011) QUADAS-2: a revised tool for the quality assessment of diagnostic accuracy studies. Ann Intern Med 155:529-536. https://doi.org/10.7326/0003-4819-155-8-201110180-00009

19. Nazarian A, Pezzella L, Tseng A, Baldassarri S, Zurakowski D, Evans CH, Snyder BD (2010) Application of structural rigidity analysis to assess fidelity of healed fractures in rat femurs with critical defects. Calcif Tissue Int 86:397-403. https://doi.org/10. 1007/s00223-010-9353-4

20. Augat P, Merk J, Genant HK, Claes L (1997) Quantitative assessment of experimental fracture repair by peripheral computed tomography. Calcif Tissue Int 60:194-199. https://doi.org/10. $1007 / \mathrm{s} 002239900213$

21. Jamsa T, Koivukangas A, Kippo K, Hannuniemi R, Jalovaara P, Tuukkanen J (2000) Comparison of radiographic and pQCT analyses of healing rat tibial fractures. Calcif Tissue Int 66:288-291. https://doi.org/10.1007/s002230010058

22. Fiset S, Godbout C, Crookshank MC, Zdero R, Nauth A, Schemitsch EH (2018) Experimental validation of the radiographic union score for tibial fractures (RUST) using micro-computed tomography scanning and biomechanical testing in an in-vivo rat model. J Bone Jt Surg Am 100:1871-1878. https://doi.org/10. 2106/jbjs. 18.00035

23. Martin Böhm A, Jungkunz B (1999) Bending stiffness of healing fractures can be calculated from quantitative computed tomography. Eur J Radiol 30:28-32. https://doi.org/10.1016/s0720048x(98)00138-7

24. Wright DA, Nam D, Whyne CM (2012) A comparison of stereology, structural rigidity and a novel 3D failure surface analysis method in the assessment of torsional strength and stiffness in a mouse tibia fracture model. J Biomech 45:2236-2240. https://doi. org/10.1016/j.jbiomech.2012.06.016

25. Sigurdsen U, Reikeras O, Hoiseth A, Utvag SE (2011) Correlations between strength and quantitative computed tomography measurement of callus mineralization in experimental tibial fractures. Clin Biomech 26:95-100. https://doi.org/10.1016/j.clinb iomech.2010.09.004

26. Nyman JS, Munoz S, Jadhav S, Mansour A, Yoshii T, Mundy GR, Gutierrez GE (2009) Quantitative measures of femoral fracture repair in rats derived by micro-computed tomography. J Biomech 42:891-897. https://doi.org/10.1016/j.jbiomech.2009.01.016

27. Morgan EF, Mason ZD, Chien KB, Pfeiffer AJ, Barnes GL, Einhorn TA, Gerstenfeld LC (2009) Micro-computed tomography assessment of fracture healing: relationships among callus structure, composition, and mechanical function. Bone 44:335-344. https://doi.org/10.1016/j.bone.2008.10.039

28. den Boer FC, Bramer JAM, Patka P, Bakker FC, Barentsen RH, Feilzer AJ, de Lange ESM, Haarman HJTM (1998) Quantification of fracture healing with three-dimensional computed tomography. Arch Orthop Trauma Surg 117:345-350. https://doi.org/10.1007/ s004020050263
29. Mehta M, Heyland M, Toben D, Duda GN (2013) Microstructure and homogeneity of distribution of mineralised struts determine callus strength. Eur Cell Mater 25:366-379. https://doi.org/10. 22203/ecm.v025a26 (discussion 378-369)

30. Shefelbine SJ, Simon U, Claes L, Gold A, Gabet Y, Bab I, Muller R, Augat P (2005) Prediction of fracture callus mechanical properties using micro-CT images and voxel-based finite element analysis. Bone 36:480-488. https://doi.org/10.1016/j.bone.2004. 11.007

31. Schwarzenberg P, Maher MM, Harty JA, Dailey HL (2019) Virtual structural analysis of tibial fracture healing from low-dose clinical CT scans. J Biomech 83:49-56. https://doi.org/10.1016/j. jbiomech.2018.11.020

32. Damron TA, Nazarian A, Entezari V, Brown C, Grant W, Calderon N, Zurakowski D, Terek RM, Anderson ME, Cheng EY, Aboulafia AJ, Gebhardt MC, Snyder BD (2016) CT-based structural rigidity analysis is more accurate than mirels scoring for fracture prediction in metastatic femoral lesions. Clin Orthop Relat Res 474:643-651. https://doi.org/10.1007/s11999-015-4453-0

33. Leong NL, Anderson ME, Gebhardt MC, Snyder BD (2010) Computed tomography-based structural analysis for predicting fracture risk in children with benign skeletal neoplasms: comparison of specificity with that of plain radiographs. J Bone Joint Surg Am 92:1827-1833. https://doi.org/10.2106/JBJS.I.00871

34. Snyder BD, Hauser-Kara DA, Hipp JA, Zurakowski D, Hecht AC, Gebhardt MC (2006) Predicting fracture through benign skeletal lesions with quantitative computed tomography. J Bone Joint Surg Am 88:55-70. https://doi.org/10.2106/JBJS.D.02600

35. Dailey HL, Schwarzenberg P, Daly CJ, Boran SAM, Maher MM, Harty JA (2019) Virtual mechanical testing based on low-dose computed tomography scans for tibial fracture: a pilot study of prediction of time to union and comparison with subjective outcomes scoring. J Bone Joint Surg Am 101:1193-1202. https://doi. org/10.2106/JBJS.18.01139

36. Allen MR (2017) Preclinical models for skeletal research: how commonly used species mimic (or don't) aspects of human bone. Toxicol Pathol 45:851-854

37. Cook SD, Patron LP, Salkeld SL, Smith KE, Whiting B, Barrack RL (2009) Correlation of computed tomography with histology in the assessment of periprosthetic defect healing. Clin Orthop Relat Res 467:3213-3220. https://doi.org/10.1007/s11999-009-1087-0

38. Gabet Y, Müller R, Regev E, Sela J, Shteyer A, Salisbury K, Chorev M, Bab I (2004) Osteogenic growth peptide modulates fracture callus structural and mechanical properties. Bone $35: 65-73$

Publisher's Note Springer Nature remains neutral with regard to jurisdictional claims in published maps and institutional affiliations. 